\title{
STABILITY OF NEURAL NETWORKS WITH RANDOM IMPULSES
}

\author{
SNEZHANA HRISTOVA ${ }^{1}$ AND PETER KOPANOV ${ }^{2}$ \\ ${ }^{1,2}$ Department of Applied Mathematics and Modeling \\ Faculty of Mathematics and Informatics \\ University of Plovdiv "Paisii Hilendarski" \\ Plovdiv, BULGARIA
}

\begin{abstract}
One of the main properties of solutions of neural networks is stability and often the direct Lyapunov method is used to study stability properties. We consider the Hopfield's graded response neural network in the case when the neurons are subject to a certain impulsive state displacement at random exponentially distributed moments. It changes significantly the behavior of the solutions because they are not deterministic ones but they are stochastic processes. We examine the stability of the equilibrium of the model. Some sufficient conditions for p-moment stability of equilibrium of neural networks with time varying self-regulating parameters of all units and time varying functions of the connection between two neurons in the network are obtained. These sufficient conditions are explicitly expressed in terms of the parameters of the system and hence they are easily verifiable. We illustrate our theory on a particular nonlinear neural network.
\end{abstract}

AMS Subject Classification: 34A37, 34D20, 34F99, 92B20

Key Words: nonlinear neural networks, random impulses, Lyapunov functions, p-moment stability

Received: May 20, 2018; Accepted: October 2, 2018;

Published: October 3, 2018 doi: $10.12732 /$ dsa.v27i4.7

Dynamic Publishers, Inc., Acad. Publishers, Ltd.

https://acadsol.eu/dsa

\section{INTRODUCTION}

The subject of artificial neural networks has become one of the important technical tools for solving a variety of problems in various scientific disciplines. From a mathematical point of view, an artificial neural network corresponds to a non-linear 
transformation of some inputs into certain outputs. Among the many types of neural networks proposed and studied in the literature, the Hopfield-type network ([5]) has become an important one due to its potential for applications in associative memory, pattern recognition, optimization, model identification, signal processing, etc. Note the stability of deterministic models with impulses for neural networks are studied in [4], [8]-[12].

A brief overview of the randomness in the neural networks and some methods for their investigations are given in [7] where the studied models are stochastic ones. Furthermore, the impulsive perturbation is a common phenomenon in real systems, so it is also an important problem how to deal with impulsive systems. Additionally, the occurrence of these impulses at random times is often particular for real system. It requires study of deterministic neural networks with impulses occurring at random time. An unsuccessful trial for studying the Hopfield neural network with impulses at random times is done by A. Vinodkumar at al. in [9]. In this paper random variables are incorrectly mixed with deterministic variables. For example, the defined $I_{\left[\xi_{k}, \xi_{k+1}\right)}(t)$ for random variables $\xi_{k}, \xi_{k+1}$ is not a deterministic index function, it is a stochastic process and it has an expected value labeled by $E$ and it has to be taken into mind in the written on page13[9]. Additionally, it is not used the well known result from probability theory that the expected value of a constant is equal to the same constant, that the expected value of a product is equal to the product of expected values only for independent random variables. So, we define for the first time the generalization of Hopfield neural network with impulses at random times, briefly give an explanation of the solutions being stochastic processes and study stability properties. Note the stability problem for differential equation with impulses at random time are studied in [1].[2]. In this paper we study the general case of Hopfield neural network with time varying self-regulating parameters of all units and time varying functions of the connection between two neurons in the network. The study is based on the application of the Lyapunov method. Using Lyapunov functions some stability sufficient criteria are provided and illustrated with examples.

\section{SYSTEM DESCRIPTION}

We will consider the model proposed by Hopfield [5] and known as Hopfield's graded response neural network in the case when the neurons are subject to a certain impulsive state displacement at random moments

Let $T_{0} \geq 0$ be a fixed point and the probability space $(\Omega, \mathcal{F}, P)$ be given. Let a sequence of independent exponentially distributed random variables $\left\{\tau_{k}\right\}_{k=1}^{\infty}$ with the same parameter $\lambda>0$ defined on the sample space $\Omega$ be given. 
Define the sequence of random variables $\left\{\xi_{k}\right\}_{k=0}^{\infty}$ by

$$
\xi_{k}=T_{0}+\sum_{i=1}^{k} \tau_{i}, \quad k=0,1,2, \ldots
$$

The random variable $\tau_{k}$ measures the waiting time of the $k$-th impulse after the $(k-1)$-st impulse occurs and the random variable $\xi_{k}$ denotes the length of time until $k$ impulses occur for $t \geq T_{0}$.

Remark 1. The random variable $\Xi=\sum_{i=1}^{k} \tau_{i}$ is Erlang distributed and it has a pdf $f_{\Xi}(t)=\lambda e^{-\lambda t} \frac{(\lambda t)^{k-1}}{(k-1) !}$ and cdf $F(t)=P(\Xi<t)=1-e^{-\lambda t} \sum_{j=0}^{k-1} \frac{(\lambda t)^{j}}{j !}$.

Consider the general model of Hopfield's graded response neural networks with impulses occurring at random times (RINN)

$$
\begin{aligned}
x_{i}^{\prime}(t) & =-c_{i}(t) x_{i}(t)+\sum_{j=1}^{n} a_{i j}(t) f_{j}\left(x_{j}(t)\right)+I_{i}(t), \\
\qquad \text { for } t \geq T_{0}, \quad \xi_{k}<t<\xi_{k+1}, k=0,1, \ldots, \quad i=1,2, \ldots n & \text { for } k=1,2, \ldots, \\
x_{i}\left(\xi_{k}+0\right) & =\Phi_{k, i}\left(x_{i}\left(\xi_{k}-0\right)\right) \quad \\
x_{i}\left(T_{0}\right) & =x_{i}^{0},
\end{aligned}
$$

where $n$ represents the number of neurons in the network, $x_{i}(t)$ is the pseudostate variable denoting the average membrane potential of the $i$-th neuron at time $t$, $x(t)=\left(x_{1}(t), x_{2}(t), \ldots, x_{n}(t)\right) \in \mathbb{R}^{n}, c_{i}(t)>0, i=1,2, \ldots, n$, is the self-regulating parameter of the $i$-th unit, $a_{i j}(t), i, j=1,2, \ldots, n$, correspond to the synaptic connection strength of the $i$-th neuron to the $j$-th neuron at time $t, f_{j}\left(x_{j}(t)\right)$ denotes the activation functions of the neurons at time $t$ and represent the response of the $j$-th neuron to its membrane potential, $f(x)=\left(f_{1}\left(x_{1}\right), f_{2}\left(x_{2}\right), \ldots, f_{n}\left(x_{n}\right)\right)$ and $I=\left(I_{1}, I_{2}, \ldots, I_{n}\right)$ is an external bias vector, the state displacements is impulsive activated at a random time equal to a value of the random variable $\xi_{k}$, the functions $\Phi_{k, i}(u), k=1,2, \ldots$ are the impulsive functions giving the impulsive perturbation of the $i$-th neuron.

\subsection{DESCRIPTION OF THE SOLUTIONS OF MODEL (2)}

Consider the sequence of points $\left\{t_{k}\right\}_{k=1}^{\infty}$ where the point $t_{k}$ is an arbitrary value of the corresponding random variable $\tau_{k}, k=1,2, \ldots$ Define the increasing sequence of points $\left\{T_{k}\right\}_{k=1}^{\infty}$ by

$$
T_{k}=T_{0}+\sum_{i=1}^{k} t_{k}
$$

Note $T_{k}$ are values of the random variables $\xi_{k}, k=1,2, \ldots$ 
Consider the initial value problem for the system of non - instantaneous impulsive differential equations with fixed points of impulses and fixed length of action of the impulses (INN):

$$
\begin{aligned}
x_{i}^{\prime}(t) & =-c_{i}(t) x_{i}(t)+\sum_{j=1}^{n} a_{i j}(t) f_{j}\left(x_{j}(t)\right)+I_{i}(t), \\
\qquad \text { for } t \geq T_{0}, \quad t \neq T_{k}, k=0,1, \ldots, \quad i=1,2, \ldots n & \text { for } k=1,2, \ldots, \\
x_{i}\left(T_{k}+0\right) & =\Phi_{k, i}\left(x_{i}\left(T_{k}-0\right)\right) \quad \\
x_{i}\left(T_{0}\right) & =x_{i}^{0},
\end{aligned}
$$

The solution of the differential equation with fixed moments of impulses (4) depends not only on the initial condition $\left(T_{0}, x_{0}\right)$ but on the moments of impulses $T_{k}, k=1,2, \ldots$, i.e. the solution depends on the chosen arbitrary values $t_{k}$ of the random variables $\tau_{k}, k=1,2, \ldots$ We denote the solution of the initial value problem (4) by $x\left(t ; T_{0}, x_{0},\left\{T_{k}\right\}\right)$. We will assume that

$$
x\left(T_{k} ; T_{0}, x_{0},\left\{T_{k}\right\}\right)=\lim _{t \rightarrow T_{k}-0} x\left(t ; T_{0}, x_{0},\left\{T_{k}\right\}\right)
$$

for any $k=1,2, \ldots$ The set of all solutions $x\left(t ; T_{0}, x_{0},\left\{T_{k}\right\}\right)$ of the initial value problem for the impulsive fractional differential equation (4) for any values $t_{k}$ of the random variables $\tau_{k}, k=1,2, \ldots$ generates a stochastic process with state space $\mathbb{R}^{n}$. We denote it by $x\left(t ; T_{0}, x_{0},\left\{\tau_{k}\right\}\right)$ and we will say that it is a solution of RINN (2).

Definition 1. For any given values $t_{k}$ of the random variables $\tau_{k}, k=1,2,3, \ldots$ respectively, the solution $x\left(t ; T_{0}, x_{0},\left\{T_{k}\right\}\right)$ of the corresponding IVP for the INN (4) is called a sample path solution of the IVP for the RINN (2).

Any sample path solution $x\left(t ; T_{0}, x_{0},\left\{T_{k}\right\}\right) \in C^{q}\left(\left(s_{k}, T_{k+1}\right], \mathbb{R}^{n}\right), k=0,1,2, \ldots$

Definition 2. A stochastic process $x\left(t ; T_{0}, x_{0},\left\{\tau_{k}\right\}\right)$ with an uncountable state space $\mathbb{R}^{n}$ is said to be a solution of the IVP for the system of RINN (2) if for any values $t_{k}$ of the random variables $\tau_{k}, k=1,2, \ldots$ the corresponding function $x\left(t ; T_{0}, x_{0},\left\{T_{k}\right\}\right)$ is a sample path solution of the IVP for RINN (2).

Definition 3. We will say that the stochastic processes $y(t)$ and $u(t)$ satisfy the inequality $y(t) \leq u(t)$ for $t \in J \subset \mathbb{R}$ if the state space of the stochastic processes $v(t)=y(t)-v(t)$ is $(-\infty, 0]$. 


\subsection{EQUILIBRIUM OF MODEL (2)}

Definition 4. A vector $x^{*} \in \mathbb{R}^{n}, x^{*}=\left(x_{1}^{*}, x_{2}^{*}, \ldots, x_{n}^{*}\right)$ is an equilibrium point of RINN (2), iff the equalities

$$
0=-c_{i}(t) x_{i}^{*}+\sum_{j=1}^{n} a_{i j}(t) f_{j}\left(x_{j}^{*}\right)+I_{i}(t) \text { for } t \geq 0, i=1,2, \ldots, n
$$

and

$$
x_{i}^{*}=\Phi_{k, i}\left(t, x_{i}^{*}, x_{i}^{*}\right) \text { for } t \geq 0, k=1,2, \ldots, i=1,2, \ldots, n
$$

hold.

We assume the following:

Assumption A1. Let the RINN (2) have an equilibrium vector $x^{*} \in \mathbb{R}^{n}$.

If assumption A1 is satisfied then we can shift the equilibrium point $x^{*}$ of system (2) to the origin. The transformation $y(t)=x(t)-x^{*}$ is used to put system (2) in the following form:

$$
\begin{aligned}
y_{i}^{\prime}(t) & =-c_{i}(t) y_{i}(t)+\sum_{j=1}^{n} a_{i j}(t) F_{j}\left(y_{j}(t)\right), \\
\text { for } t \geq T_{0}, \quad \xi_{k}<t<\xi_{k+1}, k=0,1, \ldots, \quad i=1,2, \ldots n & \text { for } k=1,2, \ldots, \\
y_{i}\left(\xi_{k}+0\right) & =\phi_{k, i}\left(y\left(\xi_{k}-0\right)\right) \quad \\
y_{i}\left(t_{0}\right) & =y_{i}^{0},
\end{aligned}
$$

where $F_{j}(u)=f_{j}\left(u+x_{j}^{*}\right)-f_{j}\left(x_{j}^{*}\right), j=1,2, \ldots, n$ and $\phi_{k, i}(u)=\Phi_{k, i}\left(u+x_{i}^{*}\right)-$ $\Phi_{k, i}\left(x_{i}^{*}\right), i=1,2, \ldots, n, k=1,2, \ldots, y_{i}^{0}=x_{i}^{0}-x_{i}^{*}$.

Note that $F_{j}(0) \equiv 0$ and according to Definition 1 the point $x^{*} \in \mathbb{R}^{n}$ is an equilibrium of RINN (2) iff $\phi_{k, i}(0) \equiv 0$, i.e. if the point $x^{*} \in \mathbb{R}^{n}$ is an equilibrium of RINN (2) then the point $y^{*}=0$ is an equilibrium of RINN (7). This allows us to study the stability properties of the zero equilibrium of of RINN (7).

\section{SOME STABILITY RESULTS FOR RANDOM IMPULSIVE DIFFERENTIAL EQUATIONS}

Consider the general type of initial value problem (IVP) for a system of nonlinear random impulsive differential equations (RIDE)

$$
\begin{aligned}
& x^{\prime}(t)=g(t, x(t)) \text { for } t \geq T_{0}, \quad \xi_{k}<t<\xi_{k+1}, \\
& x\left(\xi_{k}+0\right)=\Psi_{k}\left(x\left(\xi_{k}-0\right)\right) \quad \text { for } \quad k=1,2, \ldots \\
& x\left(T_{0}\right)=x^{0} .
\end{aligned}
$$


Definition 5. Let $p>0$. Then the trivial solution $\left(x_{0}=0\right)$ of the RIDE (8) is said to be $p$-moment exponentially stable if for any initial point $\left(T_{0}, y_{0}\right) \in \mathbb{R}_{+} \times \mathbb{R}^{n}$ there exist constants $\alpha, \mu>0$ such that $\left.E\left[\| y\left(t ; T_{0}, x_{0},\left\{\tau_{k}\right)\right\}\right) \|^{p}\right]<\alpha\left\|y_{0}\right\|^{p} e^{-\mu\left(t-T_{0}\right)}$ for all $t>T_{0}$, where $y\left(t ; T_{0}, x_{0},\left\{\tau_{k}\right)\right\}$ is the solution of the IVP for the RIDE (8).

Definition 6. Let $p>0$. Then the equilibrium $x^{*}$ of the RINN (2) is said to be $p$-moment exponentially stable if for any initial point $\left(T_{0}, x_{0}\right) \in \mathbb{R}_{+} \times \mathbb{R}^{n}$ there exist constants $\alpha, \mu>0$ such that $\left.E\left[\| x\left(t ; T_{0}, x_{0},\left\{\tau_{k}\right)\right\}\right)-x^{*} \|^{p}\right]<\alpha\left\|x_{0}-x^{*}\right\|^{p} e^{-\mu\left(t-T_{0}\right)}$ for all $t>T_{0}$, where $x\left(t ; T_{0}, x_{0},\left\{\tau_{k}\right)\right\}$ is the solution of the IVP for the RINN (2).

Remark 2. We note that the two-moment exponentially stability for stochastic equations is known as exponentially stability in mean square.

Note the p-moment exponential stability of $\operatorname{RIDE}(8)$ is studied in [3].

We will give some stability results for differential equations with non-instantaneous impulses applying Lyapunov functions from the class $\Lambda(J, \Delta), J \subset \mathbb{R}_{+}$be a given interval, and $\Delta \subset \mathbb{R}^{n}, \quad 0 \in \Delta$ be a given set:

$$
\begin{array}{r}
\Lambda(J, \Delta)=\left\{V(t, x) \in C\left(J \times \Delta, \mathbb{R}_{+}\right): V(t, 0) \equiv 0,\right. \\
V(t, x) \text { is locally Lipschitzian with respect to } \mathrm{x}\} .
\end{array}
$$

We will use the Dini derivative of the Lyapunov function $V(t, x) \in \Lambda(J, \Delta)$ along trajectories of solutions of (8) given by:

$$
\begin{aligned}
& { }_{(8)} D_{+} V(t, x)=\limsup _{h \rightarrow 0^{+}} \frac{1}{h}\{V(t, x)-V(t-h, x-h g(t, x))\} \\
& \text { for } t \in J, x \in \Delta \text {, }
\end{aligned}
$$

where for any $t \in\left(t_{k}, s_{k}\right)$ there exists $h_{t}>0$ such that $t-h \in\left(t_{k}, s_{k}\right), x-h g(t, x) \in \Delta$ for $0<h \leq h_{t}$.

Now we will give some sufficient condition that will be used further:

Theorem 1. [3]. Let the following conditions be satisfied:

1. For $t \geq 0: g(t, 0) \equiv 0$ and $\Psi_{k}(0)=0, k=1,2, \ldots$ and for any initial values $\left(T_{0}, x^{0}\right)$ the corresponding IVP for the ordinary differential equation $x^{\prime}(t)=g(t, x(t))$ has a unique solution.

2. The function $V \in \Lambda\left(\left[T_{0}, \infty\right), \mathbb{R}^{n}\right)$, and there exist positive constants $a, b$ such that

(i) $a\|x\|^{p} \leq V(t, x) \leq b\|x\|^{p}$ for $t \geq T_{0} x \in \mathbb{R}^{n}$;

(ii) there exists a function $m \in C\left(\mathbb{R}_{+}, \mathbb{R}_{+}\right)$: $\inf _{t \geq 0} m(t)=L \geq 0$ and the inequality

$$
{ }_{(8)} D_{+} V(t, x) \leq-m(t) V(t, x), \quad \text { for } t \geq 0, \quad x \in \mathbb{R}^{n}
$$


holds;

(iii) for any $k=1,2, \ldots$ there exist constants $w_{k}$ and $C$ such that $0 \leq w_{k}(t) \leq$ $C<1+\frac{L}{\lambda}$ for $t \geq 0$ such that

$$
V\left(t, I_{k}(t, x)\right) \leq w_{k} V(t, x) \quad \text { for } t \geq 0, x \in \mathbb{R}^{n} .
$$

Then the trivial solution of the RIDE (8) is p-moment exponentially stable.

\section{STABILITY ANALYSIS OF NEURAL NETWORKS WITH RANDOM IMPULSES}

We will introduce the following assumptions:

Assumption A2. The neuron activation functions are Lipschitz, i.e. there exist positive numbers $L_{i}>0, i=1,2, \ldots, n$ such that $\left|f_{i}(u)-f_{i}(v)\right| \leq L_{i}|u-v|, i=$ $1,2, \ldots, n$ for $u, v \in \mathbb{R}$.

Assumption A3. There exist positive numbers $M_{i, j}, i, j=1,2, \ldots, n$ such that $\left|a_{i, j}(t)\right| \leq M_{i, j}$ for $t \geq 0$.

Assumption A4. There exist numbers $B_{i}>0, i=1,2, \ldots, n$ such that the inequalities $c_{i}(t) \geq B_{i}>0, t \geq 0$ hold.

Assumption A5. There exists a positive inequality

$$
2 \min _{i=\overline{1, n}} B_{i}>\sum_{i=1}^{n} \max _{j} M_{i j} L_{j}+\max _{i} \sum_{j=1}^{n} M_{i j} L_{j}
$$

holds.

Assumption A6. There exists positive numbers $K_{k}$ such that for $k=1,2, \ldots$ and $x \in \mathbb{R}^{n}$ the inequalities

$$
\sum_{i=1}^{n}\left(\Phi_{k, i}\left(x_{i}\right)-\Phi_{k, i}\left(x_{i}^{*}\right)\right)^{2} \leq K_{k} \sum_{i=1}^{n}\left(x_{i}-x_{i}^{*}\right)^{2},
$$

hold where $x^{*}$ is the equilibrium point from assumption A1.

Remark 3. In the case the self-regulating parameters of the $i$-th unit in the system (2) are constants Assumption A4 is satisfied.

Remark 4. If assumption A4 is fulfilled then the function $F$ in RINN (7) satisfies $\left|F_{j}(u)\right| \leq L_{j}|u|, j=1,2, \ldots, n$ for any $u:|u| \leq \lambda$.

Remark 5. If assumption $\mathrm{A} 6$ is fulfilled then the impulsive functions $\phi_{k}, k=$ $1,2, \ldots$ in RINN (7) satisfy the inequalities $\sum_{i=1}^{n} \phi_{k, i}^{2}\left(u_{i}\right)=\sum_{i=1}^{n}\left(\Phi_{k, i}\left(u_{i}+x_{i}^{*}\right)-\right.$ $\left.\Phi_{k, i}\left(x_{i}^{*}\right)\right)^{2} \leq \sum_{i=1}^{n} u_{k}^{2}$. 
Theorem 2. Let assumptions A1-A6 be satisfied.

Then the equilibrium point $x^{*}$ of RINN (2) is p-moment exponentially stable.

Proof. Apply the quadratic Lyapunov function $V(t, x)=x^{T} x$ to the system (7) and for any $t \in\left[0, s_{1}\right] \cup_{k=1}^{\infty}\left[t_{k}, s_{k+1}\right]$ and $y \in S(\lambda)$ we have

$$
\begin{aligned}
{ }_{(7)} D_{+} V(t, y) \leq & -2 \sum_{i=1}^{n} c_{i}(t) y_{i}^{2}+2 \sum_{i=1}^{n} \sum_{j=1}^{n}\left|a_{i j}(t)\right|\left|F_{j}\left(y_{j}\right)\right| y_{i} \mid \\
\leq & -2 \min _{i=\overline{1, n}} a B_{i} \sum_{i=1}^{n} y_{i}^{2}+\sum_{i=1}^{n} \sum_{j=1}^{n} M_{i j} L_{j}\left(y_{j}^{2}+y_{i}^{2}\right) \\
\leq & -2 \min _{i=1, n} B_{i} \sum_{i=1}^{n} y_{i}^{2}+\sum_{i=1}^{n} \sum_{j=1}^{n} M_{i j} L_{j} y_{j}^{2}+\sum_{i=1}^{n} \sum_{j=1}^{n} M_{i j} L_{j} y_{i}^{2} \\
\leq & -2 \min _{i=1, n} B_{i} \sum_{i=1}^{n} y_{i}^{2}+\left(\sum_{i=1}^{n} \max _{j} M_{i j} L_{j}\right) \sum_{j=1}^{n} y_{j}^{2} \\
& +\sum_{i=1}^{n} y_{i}^{2}\left(\max _{i}\left(\sum_{j=1}^{n} M_{i j} L_{j}\right)\right. \\
= & -\left(2 \min _{i=\overline{1, n}} B_{i}-\sum_{i=1}^{n} \max _{j} M_{i j} L_{j}-\max _{i} \sum_{j=1}^{n} M_{i j} L_{j}\right) \sum_{i=1}^{n} y_{i}^{2} .
\end{aligned}
$$

Define

$$
m(s) \equiv 2 \min _{i=1, n} B_{i}-\sum_{i=1}^{n} \max _{j} M_{i j} L_{j}-\max _{i} \sum_{j=1}^{n} M_{i j} L_{j} .
$$

Then condition 2(ii) of Theorem 1 is satisfied.

Also, from A8 it follows the condition 2(iii) of Theorem 1 is satisfied.

From Theorem 1 the zero solution of the system (8) is p-moment exponentially stable and therefore the equilibrium point $x^{*}$ of RINN (2) is p-moment exponentially stable.

EXAMPLE 1. Let $n=3, t_{0}=0, s_{k}=\left(k-0.5^{k}\right) \pi, t_{k}=k \pi, k=1,2, \ldots$ Consider the following partial case of the neural network with impulses at random times (2):

$$
\begin{aligned}
& x_{i}^{\prime}(t)=-(1+|\csc (t)|)\left(x_{i}(t)-0.5 \pi\right)+\sum_{j=1}^{3} a_{i j}(t) \cos \left(x_{j}(t)\right) \text {, } \\
& \text { for } t \geq 0 \quad \xi_{k}<t<\xi_{k+1}, \quad i=1,2,3 \\
& x_{i}\left(\xi_{k}+0\right)=\Phi_{k, i}\left(x_{i}\left(\xi_{k}-0\right)\right) \quad \text { for } k=1,2, \ldots \\
& x_{i}(0)=x_{i}^{0},
\end{aligned}
$$

with $\Phi_{k, i}(u)=\sin t_{k} u+\left(1-\sin t_{k}\right) 0.5 \pi, \tau_{k} \in \operatorname{Exp}(1)$, i.e. $\lambda=1$ and $A=a_{i j}(t)$ is 
given by

$$
A(t)=\left(\begin{array}{ccc}
-0.1 \sin t & 0.4 & 0.3 \\
-\frac{t^{2}}{5 t^{2}+1} & 0.3 & \frac{t}{5 t+1} \\
\frac{t}{10 t+1} & -0.2 \cos t & -0.1 \sin t
\end{array}\right) .
$$

The point $x^{*}=(0.5 \pi, 0.5 \pi, 0.5 \pi)$ is the equilibrium point of RINN (13).

Note assumptions A2 is satisfied with $L=1$.

Also assumption A4 is satisfied with $B=1$.

Next assumption A6 is fulfilled with $K_{k}=1$ because

$$
\begin{aligned}
\sum_{i=1}^{3}\left(\Phi_{k, i}\left(x_{i}\right)-\Phi_{k, i}\left(x_{i}^{*}\right)\right)^{2} & =\sum_{i=1}^{3}\left(\sin t_{k} x_{i}+\left(1-\sin t_{k}\right) 0.5 \pi-0.5 \pi\right)^{2} \\
& =4 \sum_{i=1}^{3}\left(\sin t_{k}\left(x_{i}-0.5 \pi\right)\right)^{2} \\
& \leq \sum_{i=1}^{3}\left(x_{i}-0.5 \pi\right)^{2}, k=1,2, \ldots
\end{aligned}
$$

$C: 0<w_{k} \leq C<1+\frac{L}{\lambda}=2$

Also, $\left|a_{i j}\right| \leq M_{i j}, i, j=1,2,3, t \geq 0$ where $M=\left\{M_{i j}\right\}$, is given by

$$
M=\left(\begin{array}{lll}
0.1 & 0.4 & 0.3 \\
0.2 & 0.3 & 0.2 \\
0.1 & 0.2 & 0.1
\end{array}\right)
$$

and $(0.4+0.3+0.2)+\max (0.8,0.7,0.4)<2$, i.e. the assumption A5 is fulfilled.

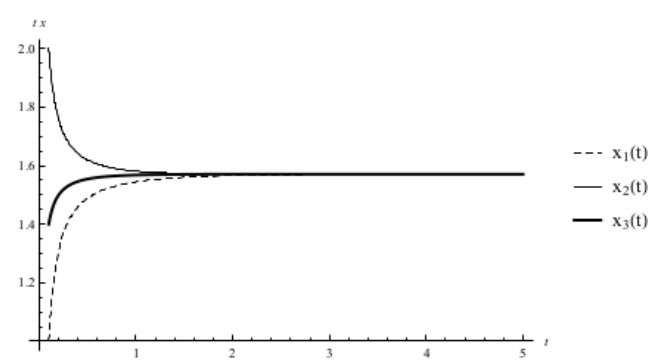

Figure 1: Example 1. Graph of the solution of the system ODE.

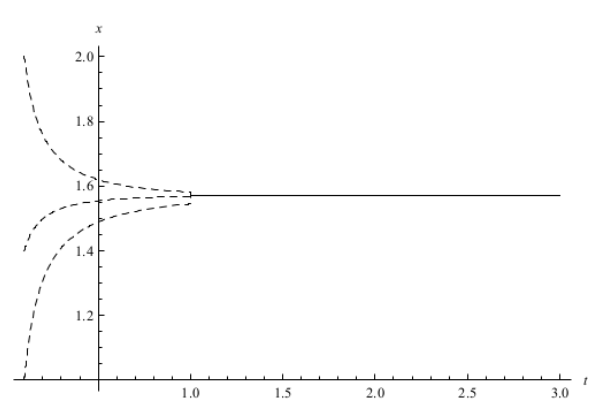

Figure 2: Example 1. Graph of the solution of RINN (13) with an impulse at 1 .

Therefore, according to Theorem 1 the equilibrium of RINN (13) is exponentially stable in mean square. 
Consider the system (13) without any kind of impulses. The equilibrium $x^{*}=$ $(0.5 \pi, 0.5 \pi, 0.5 \pi)$ is asymptotically stable (see Figure 2 and Figure 3 ). Therefore, the appropriate perturbing of the neural networks by impulses at random time can keep the stability properties of the equilibrium.

\section{ACKNOWLEDGEMENTS}

Research was partially supported by Fund PP18-FMI-004, University of Plovdiv "Paisii Hilendarski".

\section{REFERENCES}

[1] R. P. Agarwal, S. Hristova, D. O'Regan, P. Kopanov, p-moment exponential stability of differential equations with random impulses and the Erlang distribution, Mem. Diff. Eq,d Math. Phys., 70 (2017),99-106.

[2] R. P. Agarwal, S. Hristova, D. O'Regan, P. Kopanov, Impulsive differential equations with Gamma distributed moments of impulses and p-moment exponential stability, Acta Math. Sci., 37 , No. 4 (2017), 985-997

[3] R. Agarwal, S. Hristova, D. O'Regan, Exponential stability for differential equations with random impulses at random times, Adv. Diff. Eq., 2013 (2013), 372, $12 \mathrm{p}$.

[4] K. Gopalsamy, Stability of artificial neural networks with impulses, Appl. Math. Comput., 154 (2004), 783-813.

[5] J.J. Hopfield, Neural networks and physical systems with emergent collective computational abilities, Proc. Nat. Acad. Sci. USA, 79 (1982), 2554-2558.

[6] R. Rakkiyappan, P. Balasubramaiam, J. Cao, Global exponential stability of neutral-type impulsive neural networks, Nonlinear Anal. Real World Appl., 11 (2010), 122-130.

[7] S. Scardapane, D.Wang, Randomness in neural networks: An overview, WIREs Data Mining Knowl. Discov., 7 (2017), 1-18.

[8] X. Song, P. Zhao, Z. Xing, J. Peng, Global asymptotic stability of CNNs with impulses and multi-proportional delays, Math. Methods Appl. Sci., 39 (2016), 722-733.

[9] A. Vinodkumar, R. Rakkiyappan, Exponential stability results for fixed and random type impulsive Hopfield neural networks, Int. J. Comput. Sci. Math., 7, No. 1 (2016), 1-19. 
[10] Z. Wu, Ch. Li, Exponential stability analysis of delayed neural networks with impulsive time window, Advanced Computational Intelligence (ICACI), 2017 Ninth International Conference on, 2017, 37-42.

[11] Z. Yang, D. Xu, Stability analysis of delay neural networks with impulsive effects, IEEE Transactions on Circuits and Systems II: Express Briefs , 52, No. 8, (2015), 517-521.

[12] Q. Zhou, Global exponential stability of BAM neural networks with distributed delays and impulses, Nonlinear Anal. Real World Appl., 10 (2009), 144-153. 
